\title{
Research on Crack Discontinuous Interface Simulation Method Based on the Mesh Free Diffraction Criterion
}

\author{
Yuantao SUN*, Zengzeng ZHANG**, Qing ZHANG***, Xianrong QIN**** \\ *School of Mechanical Engineering, Tongji University, Shanghai, China, E-mail: sun1979@ sina.com \\ **School of Mechanical Engineering, Tongji University, Shanghai, China, E-mail: zhangzz94@ 126.com \\ ***School of Mechanical Engineering, Tongji University, Shanghai, China, E-mail: zhqing_tj@126.com \\ ****School of Mechanical Engineering, Tongji University, Shanghai, China, E-mail: tjqin@tongji.edu.cn \\ cross $^{\text {ref }}$ http://dx.doi.org/10.5755/j01.mech.24.3.19009
}

\section{Introduction}

Most fracture mechanics problems in engineering are solved by numerical analysis method such as finite element method (FEM) or boundary element method [1-2], because only few simple and special fracture mechanics problems can be solved analytically.

Mesh free method is a new numerical method that appeared in recent years. Mesh free method discretizes the entire solution domain to independent nodes instead of connecting nodes into the elements, so that mesh free method overcomes the defects of the finite element method which need re-mesh the grid continuously when it is used to solve the dynamic discontinuous boundaries problem such as crack propagation. Without elements constraints, mesh free method can conveniently track the crack propagation by adding removable refinement nodes on the crack tip area [3]

However, the mesh free method still has some defects such as the modeling of crack discontinuous interface in practical. In general, it is easy to model the crack discontinuity line through the element boundary in FEM. But mesh free method only has node so that it can't model such crack element interface similar with FEM. At the same time, as a new method in development, its rigorous mathematical derivation and computational efficiency is still behind the FEM. When it used to deal with discontinuous interface problems such as crack, its base function, weight function argument and approximate function etc. are not mature, which are mainly determined by experience [3]. At present, there are mainly three criterions to simulate the discontinuity line in the mesh free method: visibility criterion, diffraction criterion and transparency attenuation criterion [4]. According to literatures, the most used method is diffraction method, but while visibility method and transparency attenuation method is less used. The diffraction criterion [5] is proposed by Belyschko, Organ, etc., that and this idea comes from the physical phenomenon which that the light occurs diffraction when it encounters a sharp point. In the diffraction phenomenon, discontinuous line is still regarded as opaque, but the "light" from the node can bypass the cusp of discontinuous lines. In order to simulate the diffraction effect including visibility method and transparency attenuation in crack problem, the equivalent distance as weight function argument need to be calculated, which is also the main strategy of mesh free method to simulate the influence of crack.

The paper firstly describes the basic principle of the mesh free method and process of the calculation process of weight function. Based on the original diffraction criterion, a new function is derived and put forward, which the binary linear equation is used to calculate the equivalent distance for the weight function arguments to dispose of the crack's discontinuous interface. The new method can simulate the displacement field around crack tip or crack closure line and also avoid the value overflow phenomenon which the node and a computation superposition occurs in the present processing method. Finally, the stress intensity factors of three typical crack are calculated combining the displacement extrapolation method as example. The comparison with the analytical solution shows that their stress intensity factors have a better precise based on the new function according to the diffraction criterion, which also verified the method valid.

\section{The basic principles of the mesh free method}

Basic equation for solving domain $\Omega$ [6]

$$
\begin{cases}\nabla \sigma+f=0 & \text { in } \Omega, \\ u=\bar{u} & \text { in } \Gamma_{u}, \\ \sigma n=\bar{t} & \text { in } \Gamma_{t} .\end{cases}
$$

Where: $\Gamma_{u}, \Gamma_{t}$ is the given displacement boundary condition; $\Gamma_{u}, \Gamma_{t}$ is the given surface force boundary condition, $f$ refers to the given volume force, $\nabla$ is called differential operator, $n$ is the cosine of the outer normal direction; $\bar{t}, \bar{u}$ are the surface force and the displacement vector of the given boundary, $u$ is the displacement vector of any point in the domain $\Omega, \sigma$ is the stress tensor corresponding to $u$, according to the linear elastic conditions:

$$
\left\{\begin{array}{l}
\varepsilon=\Delta_{t} u, \\
\sigma=D \varepsilon,
\end{array},\right.
$$

In Eq. (2), $\varepsilon$ is the strain:

$$
D=\frac{E}{1-\mu^{2}}\left[\begin{array}{ccc}
1 & \mu & 0 \\
\mu & 1 & 0 \\
0 & 0 & (1-\mu) / 2
\end{array}\right]
$$

By Eq. (1), the equivalent weak integral form can be got such as Eq. (4)[4]: 


$$
\begin{aligned}
\delta \prod(u) & =\int_{\Omega}\left(\delta(\nabla s \cdot u)^{T} \sigma-\delta u^{T} f\right) d \Omega- \\
& -\int_{\Gamma_{t}} \delta u^{T} \bar{t} d \Gamma-\int_{\Gamma_{u}} \delta \lambda^{T}(u-\bar{u}) d \Gamma-, \\
& -\int_{\Gamma_{u}} \delta u^{T} \lambda d \Gamma=0 .
\end{aligned}
$$

Where $\delta$ is non-variation operator, $\nabla_{s}$ is symmetric gradient operator. Because it cannot be solved complex problems accurately, the targets of problem-solving is to get the nodal displacement that can satisfy the boundary conditions. In the mesh free method, the function value of the nodes $x_{I}(I=1,2, . ., N ; N$ is the number of node $)$ in the solving domain $u^{*}=u\left(x_{I}\right)$ are assumed to be known, thus the global approximation function $u(x)$ constructed in the solving domain is $u^{h}(x)$, for the calculation point $x$ (usually Gaussian points), the unknown function $u(x)$ is approximately as follows:

$$
u \approx u^{h}(x)=\sum_{i=1}^{m} p_{i}(\bar{x}) a_{i}(x)=p^{T}(\bar{x}) a(x) .
$$

Where: $\bar{x}=[x, y, z]^{T}$ is the spatial coordinates of all points in the neighborhood of the calculation point $x$, $p^{T}(\bar{x})=\left[p_{1}(\bar{x}), p_{2}(\bar{x}), \ldots, p_{m}(\bar{x})\right], \quad p_{i}(\bar{x})$ is the primary function, $m$ is the number of the primary functions, which the generally used primary function include linear base and square base:

$$
\begin{aligned}
& p^{T}(\bar{x})=[1, x, y] m=3 \text { linear base, } \\
& p^{T}(\bar{x})=\left[1, x, y, x^{2}, x y, y^{2}\right] m=6 \text { square base. }
\end{aligned}
$$

In the mesh free method, the global displacement approximation function can be constructed by the moving least square approximation (Moving Least Square Approximation MLSA) [5].

For the Eq. (5): $a(x)=\left[a_{1}(x), a_{2}(x), \ldots, a_{m}(x)\right]^{T}$, $a_{i}(x)$ is the undetermined coefficient, it ensures that the approximate function $u^{h}(x)$ is the best approximation in the neighborhood of calculation point $x$ in the least-squares sense. So we define weighted quadratic sum of the approximate function's error in calculation point $x$ is:

$$
\begin{aligned}
J(x) & =\sum_{i=1}^{N} w\left(x-x_{i}\right)\left[u\left(x_{i}\right)-u_{i}\right]^{2}= \\
& =\sum_{i=1}^{N} w\left(x-x_{i}\right)\left[\sum_{i=1}^{m} p_{i}(x) a_{i}(x)-u_{i}\right]^{2}
\end{aligned}
$$

Where $w\left(x-x_{I}\right)$ is the weight function, it is a nonnegative function with compact supporting, $w\left(x-x_{I}\right) \neq 0$ when the nodes are outside the influence domain, it has a great influence on the smoothness of field function and the convergence speed of the calculation results etc. The selection of the weight function generally should have the following principles $[7,8]$ :

The value of the weight function must be non-negative;

The coefficient $a(x)$ is unique, i.e. A-1 (x) exists;

The value of the weight function is larger when the point close to $x$;

Except for above conditions, there is no theoretical rules for the determination of the weight function's specific form. Currently commonly used weight function have
Gaussian weight function, the quartic spline function [9],in the paper the exponential weight function [10] are adopted, that it is used to the crack problem usually.

$$
w\left(x-x_{I}\right)= \begin{cases}e^{-(r \alpha)^{2}} & r \leq 1, \\ 0 & r>1 .\end{cases}
$$

Where: $r=s_{0}(x) / r_{m}, s_{0}(x)$ is the distance between the node and the calculation point, $r_{m}$ is the radius of the rounded influence domain of the node, and the influence domain may also be rectangular. Through Eq. (7), the distance between the calculation point and node has a great influence on weight function. Therefore, the distance between the calculation point and a node is the key parameter of the weight function.

The parameters $a j(x)$ are found by minimizing the quadratic functional $J(x)$ :

$$
\begin{aligned}
& \frac{\partial J}{\partial a_{j}(x)}=2 \sum_{I=1}^{N} w\left(x-x_{I}\right) \times \\
& \times\left[\sum_{I=1}^{N} p_{i}\left(x_{I}\right) a_{i}(x)-u_{I}\right]^{2} p_{i}\left(x_{I}\right)=0 .
\end{aligned}
$$

Where: $j=1,2, \ldots, m$, thus:

$$
\begin{aligned}
& a(x)=A^{-1}(x) B(x) u^{*}, \\
& A(x)=p^{T} w p \quad B(x)=p^{T} w \\
& u(\bar{x}, x)=p^{T}(x) A^{-1}(x) B(x) u .
\end{aligned}
$$

We can define that $\Phi(x)=p^{T}(x) A^{-1}(x) B(x), \Phi(x)$ is also called shape function [11].

$$
u^{h}(x)=\Phi(x) u^{*}
$$

Eq. (13) eventually is deduced based on Eq. (4):

$$
\left[\begin{array}{cc}
K & G \\
G^{T} & 0
\end{array}\right]\left[\begin{array}{c}
u^{*} \\
\bar{\lambda}
\end{array}\right]=\left[\begin{array}{l}
P \\
q
\end{array}\right] .
$$

Eq. (13) can be solved to give the displacement fitting parameters $u^{*}$, where:

$$
\begin{aligned}
& K=\int_{\Omega} B^{T} D B d \Omega, \\
& G=-\int_{\Gamma_{u}} \Phi^{T}(x) D B d \Gamma, \\
& P=\int_{\Omega} \Phi^{T}(x) f d \Gamma+\int_{\Gamma_{t}} \Phi^{T}(x) \bar{t} d \Gamma, \\
& q=\int_{\Gamma_{t}} N^{T} \bar{u} d \Gamma, \\
& B=\left[B_{1}, B_{2}, \ldots, B_{I}, \ldots B_{N}\right] .
\end{aligned}
$$

Where: $B_{I}$ is given by Eq. (19): 


$$
B_{I}=\left[\begin{array}{cc}
\Phi_{I, x} & 0 \\
0 & \Phi_{I, y} \\
\Phi_{I, y} & \Phi_{I, x}
\end{array}\right] .
$$

So the displacement value in the entire solution domain is given by Eq. (12), then we can obtain the function expression of the stress value in the whole solution domain.

$$
\sigma=D B u^{*}
$$

\section{The analysis of mesh free crack based on diffraction criterion}

\subsection{Diffraction criterion}

The finite element method is relatively simple to handle discontinuous problems such as crack, because it sets the element boundary directly at the discontinuity line, thus the displacement function interpolation, energy functional integral is restricted in the element. While the mesh free method is difficult, the shape function is based on the fit values of the node displace within the solution domain. When encounter discontinuous problems such as cracks, mesh free method usually reflects this discontinuity by three principles: visibility criterion, diffraction criterion, transparent attenuation criterion.

Visibility criterion [12] is the easiest way to introduce discontinuities; it assumed that in the solution domain the connections line from the calculation point to the node can be seen as a bunch of light, and if this connection line intersects with discontinuous lines, then the calculation point is supposed to be excluded from the node influence of domain. This rule relatively is rough, it may lead to unreal intermittent of the shape function in the tip of discontinuous line, and is not a good analog for non-convex boundary.

Transparent attenuation criterion [13-15] consider discontinuous line with a certain transparency, and the discontinuous cusp points is treated as completely transparent, thereby eliminating the discontinuity; the rest discontinuous lines transparency decreases with the Cusp distance, shown in Fig. 1, i.e.

Due to the discontinuous line barrier, the distance parameter of the weight function can't be directly defined as the distance $s_{0}(x)$ between the node and the calculation point by conventional methods, but need to be modified to an equivalent distance $s(x)$, which may affect the interaction strength of the calculation point and node. In the Transparent attenuation criterion, the equivalent distance $s(x)$ is expressed as Eq. (21) witch substituting its value into the weight function expression can obtain the calculation nodes' weights.

$$
s(x)=s_{0}(x)+s_{\max }\left(\frac{s_{c}(x)}{\overline{s_{c}}(x)}\right)^{\lambda} .
$$

Where: $s_{\max }$ in the Eq. (21) is the radius of the influence domain of nodes. $s_{c}(x)$ is the distance from crack tip to intersection of the "light" and the crack. Define the parameters $\overline{s_{c}}$ as $\overline{s_{c}}=\kappa h$, where the constant $\kappa$ is used to adjust the transparency (usually in the range between 0 and $1), h$ is the average distance of nodes.

In the transparency method, if the node is too close to the crack line, the angle between the node-crack tip connection with the crack itself will be very small, that means $\overline{s_{c}}$ changes dramatically, which lead to the approximate function has a very steep gradient of. So the transparency method requires nodes not be too close to the crack. In general the vertical distance between nodes and the crack should be greater than $\kappa h$ [16].



Fig. 1 The transparency attenuation method

As mentioned, Belyschko, Organ, etc. proposed the diffraction criterion to describe the crack discontinuous lines. As shown in Fig. 2, the equivalent distance $s(x)$ between node $x_{I}$ in weight function and point $x$ is given by the following formula:

$$
s(x)=\left(\frac{s_{1}+s_{2}(x)}{s_{0}(x)}\right)^{\lambda} s_{0}(x),
$$

where: $s_{0}(x)=\left\|x-x_{I}\right\|, s_{1}(x)=\left\|x_{c}-x_{I}\right\|, s_{2}(x)=\left\|x_{c}-x\right\|$ the specific definition of the distances is as shown in Fig. 2.

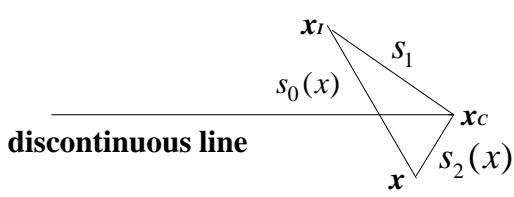

Fig. 2 Diffraction method

$x c$ is crack tip. $\lambda$ is a constant, literature [17] studied its effect to $s(x)$, who's the conclusion consider that the $\lambda$ value between 1 to 2 is more appropriate.

According to the Eq.(22) when $\lambda>1$ and $s_{1}+s_{2}(x)$ is a constant, the $s(x)$ becomes larger with the decrease of $s_{0}$, that means if other conditions remain unchanged, the equivalent distance will be longer.

\subsection{New function based on diffraction criterion}

According to the criterion of crack simulation, it can be found that the equivalent distance mainly reflects the length relation between $s_{1}$ (from calculation point to the crack tip), $s_{2}$ (from the node to the crack tip), $s_{0}$ (calculation point and the node). Intuitively, the longer $s_{1}+s_{2}$ is, the longer the distance of the light goes around the tip is, so that the weaker the influence between the calculation point and node. Therefore, this paper argues that as long as the law is satisfied, its function type should be varied.

In addition, according to the current criterion, the refinement nodes perhaps are arranged on the cracked interface and the crack tip. If the calculated point $x$ and the node $x_{I}$ coincides, then the denominator in Eq. (22) is zero, and the program occurs numeric overflow error. Especially, in 
the process of crack propagation analysis, the simulation usually cannot be carried out because the calculated point $x$ and the node $x_{I}$ coincides when it is necessary to reconstruct each crack state and refinement nodes are arranged on the crack tip. Therefore, a new function is constructed based on original diffraction criterion, and the derivation process is as follows: on both sides of the logarithm of equation (22), we obtain:

$$
\ln s(x)=\lambda \ln \left(s_{1}+s_{2}(x)\right)-(\lambda-1) \ln s_{0}(x) .
$$

Eq. (23) shows the logarithmic form of Eq. (22). To simplify calculation, this paper proposes a new function type which uses an equivalent distance expression in weight function, a binary linear equation, to deal with the crack discontinuous line. the equivalent distance is in the form of this:

$$
s(x)=A\left(s_{1}+s_{2}(x)\right)-(A-1) s_{0}(x) .
$$

It can be seen that the Eq. (24) has similar properties with Eq. (22): equivalent distance will increase with the decrease of $s_{0}$ or the increase of $s_{1}+s_{2}(x)$ when other conditions remain unchanged. Compared with Eq. (22), the Eq. (24) has an advantage that it can directly calculate the case $s_{0}=0$, whereas, when the denominator in Eq. (22) is zero, the program often occurs numeric overflow error. After testing, when $A=3.1$, Eq. (24) can make the results of method with high accuracy.

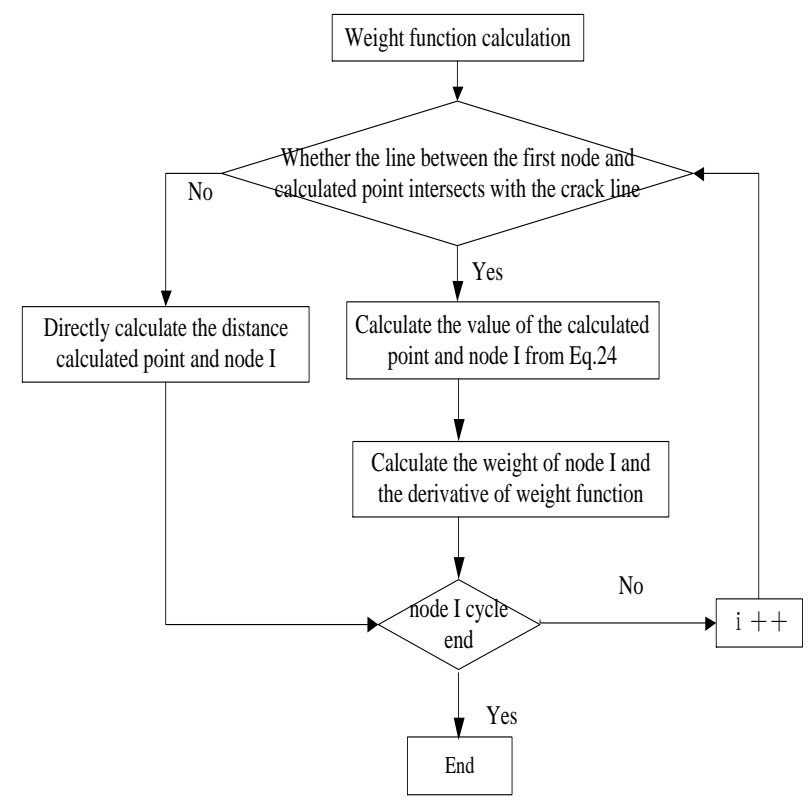

Fig. 3 Calculation process of the cracked structure weight function

\section{Verification of new method}

Considering that the mostly mechanical mode of cracks in the project is composed of tension, bending and concentrated force, the three typical crack theoretical models are established in this paper. In order to verify above conclusions, this paper calculates analytical solution of the crack model firstly, then takes analysis and verifies the conclusions.
4.1. Case 1: single edge crack specimen under uniform tension at both ends

The single edge crack specimen is shown as Fig. 4, its length $L=800 \mathrm{~mm}$, width $b=100 \mathrm{~mm}$, thickness $B=1 \mathrm{~mm}$, the remote stress of structure is $50 \mathrm{MPa}$, crack length is $10 \mathrm{~mm}$, which locates in the specimen center.

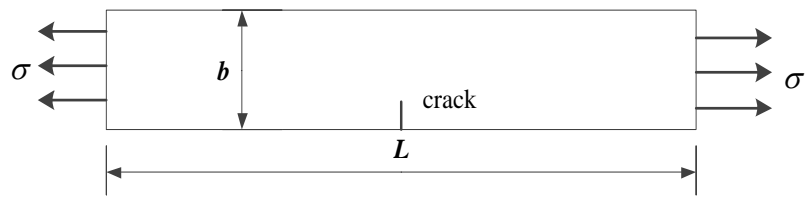

Fig. 4 The both side was tensioned

SIF is the key parameter to characterize the stress and strain fields around the crack tip, as originally described by Irwin, SIF plays a dominant role because it indicates the singular intensity of the crack field.

According to the manual of stress intensity factor, the analytical solution of the stress intensity factor of the structure is as following:

$$
K_{I}=Y \sigma \sqrt{\pi a},
$$

where the shape function $Y[18]$ is:

$$
\begin{aligned}
Y= & 1.12-0.231\left(\frac{a}{b}\right)+10.55\left(\frac{a}{b}\right)^{2}- \\
& -21.72\left(\frac{a}{b}\right)^{3}+30.39\left(\frac{a}{b}\right)^{4} .
\end{aligned}
$$

So according to Eq. (26) and Eq. (27): $K_{I}=331.7 \mathrm{MPa} \sqrt{\mathrm{mm}}$.

In this paper, matlab program for mesh free method analysis of crack is completed as there is no special software for mesh free computing. The program is made up of 7 parts; these are discrete function disnode() which create nodes in solving domain, weight function weight(), shape function shape(), stiffness function kmatrix(), boundary applied function bondary(), displacement solution of function disu() and the stress intensity factor function sif().

For this structure, with disnode(), this model is arranged uniformly 891 discrete nodes, which the distance between adjacent nodes is $10 \mathrm{~mm}$, except the distance between two nodes of the crack line is 0 . Thus the entire structure is divided into $80 \times 10$ sub-domains of integration, 36 Gaussian nodes are in each sub-domain of integration. The nodes distribution scheme is shown as Fig. 5. Then the crack tip nodes are refinement by circled shape, which the refinement nodes are arranged every 45 degrees on the circumferential direction; on radial direction, the nodes are arranged on the location where the distance to crack tip node is 1,2,3,4 mm respectively. As shown in Fig. 6, the number of the refinement nodes is 36 , so the total node number is 927. The form of primary function is linear primary functions, once the node coordinates and the node number are obtained, the calculation on the shape function and the weight function will also be conducted no longer be joined into elements, the preparation work of data is much more concise than the finite element. 


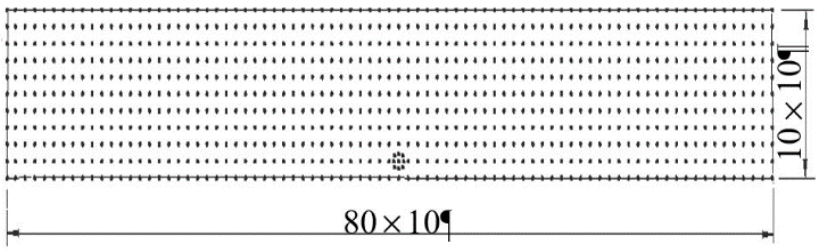

Fig. 5 Distribution scheme



Fig. 6 Node encryption of crack tip

Then the weight function of each Gaussian node is calculated by the function weight () according to mesh free method. The connection line between nodes and Gaussian nodes may deal with the crack line owning to that there are cracks in structures; there is a new problem which we need to take account for: firstly, we should check if the connection line between the Gaussian node and node I intersects with the crack line; if not, we may substitute the distance between the two nodes into Eq. (7) to obtain the weight function directly, otherwise the equivalent distance will be obtained in Eq. (24). Till the calculation process loops through all the nodes which are in the influence domain of Gaussian nodes.

After that all nodes' displacement are obtained, the stress intensity factor of the crack tip can also be calculated by the function sif(), that which is to obtain the displacement of the nodes on the crack line and the distance between the nodes on the crack tip, and we can get the stress intensity factor (SIF) result by mesh free method based on displacement extrapolation method [19]:

$K_{I}=330.3 \mathrm{MPa} \sqrt{\mathrm{mm}}$.

\subsection{Case 2: a pair of concentrated normal forces on the} crack tip

In order to deal with the discontinuity line of the crack structure in mesh free method with the higher precision.

The boundary condition of the Fig. 7 is taken as a pair of concentrated normal forces on the crack tip, namely $\mathrm{P}=1000 \mathrm{~N}$. The other does not change is shown in Fig. 7 .

The analytical solution for the stress intensity fac-

tor is:

$$
K_{I}=Y 2 P / \sqrt{\pi a},
$$

where the shape function $Y[18]$ is:

$$
\begin{aligned}
& Y=\frac{3.52}{\left(1-\frac{a}{b}\right)^{\frac{3}{2}}}-\frac{4.35}{\left(1-\frac{a}{b}\right)^{\frac{1}{2}}}+ \\
& +2.13\left(1-\frac{a}{b}\right)=1.45437 .
\end{aligned}
$$

So according to Eq. (28) and Eq. (29): $K_{I}=519.1 \mathrm{MPa} \sqrt{\mathrm{mm}}$.

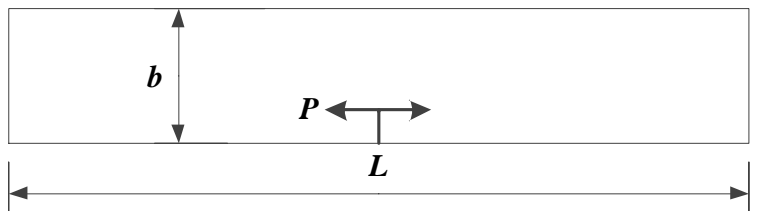

Fig. 7 A pair of concentrated normal forces on the crack tip

When the meshfree method is used, the layout scheme, the node encryption criterion and so on are equal to the both side was tensioned, and only the loading position is changed. The results can be calculated by program:

$K_{I}=509.3 \mathrm{MPa} \sqrt{\mathrm{mm}}$.

\subsection{Case 3: under pure bending condition}

The boundary condition of the Fig. 4 is taken as under pure bending condition and its force situation is shown in Fig. 8.

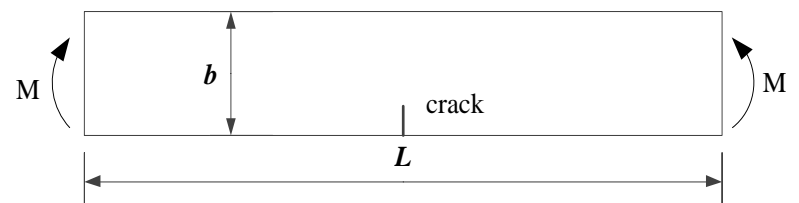

Fig. 8 Under pure bending condition

The analytical solution for the stress intensity factor is:

$$
K_{I}=Y 6 M \sqrt{\pi a} / b^{2}
$$

where the shape function $Y[18]$ is:

$$
\begin{aligned}
& Y=1.122-1.40\left(\frac{a}{b}\right)+7.33\left(\frac{a}{b}\right)^{2}- \\
& -13.08\left(\frac{a}{b}\right)^{3}+14\left(\frac{a}{b}\right)^{4}=1.044 .
\end{aligned}
$$

So according to Eq. (30) and Eq. (31): $K_{I}=491.2 \mathrm{MPa} \sqrt{\mathrm{mm}}$.

When the mesh free method is used, the layout scheme, the node encryption criterion and so on are equal to the both side was tensioned, and only the loading position is changed. The results can be calculated by program: $K_{I}=480.4 \mathrm{MPa} \sqrt{\mathrm{mm}}$. 


\subsection{Result comparison}

The above results are shown in Table 1. Through the comparison of the analytical results of above problems, the Eq. (24) as a new function can be considered to deal with the discontinuity line of the crack structure in mesh free method with the higher precision.

Table 1

Comparison of the results of three kinds of stress cases ( $\mathrm{MPa} \sqrt{\mathrm{mm}}$ )

\begin{tabular}{|l|l|l|l|}
\hline Computing Method & Case 1 & Case 2 & Case 3 \\
\hline Analytic Method & 331.7 & 519.1 & 491.2 \\
\hline Mesh free Method & 330.3 & 509.3 & 480.4 \\
\hline
\end{tabular}

\section{Conclusions}

Because mesh free method discretizes the entire solution domain to independent nodes instead of connecting nodes into the elements, the method which deals with the crack discontinuity line by using binary linear equation as the weight function's independent variable is presented based on the mesh free diffraction criterion. Through comparison of analytical results of different crack problems, it is proved that equations and chosen parameters are proper and stress intensity factor can be calculated precisely, that which also verified the method valid. Also, this method avoids the numerical overflow error which may occur when the node coincides with the calculated point in the processing.

\section{References}

1. Bouchard, P.O.; Bay, F.; Chastel, Y. 2003. Numerical modelling of crack propagation: Automatic remeshing and comparison of different criteria, Computer Methods in Applied Mechanics and Engineering 192(35-36): 3887-3908. https://doi.org/10.1016/S0045-7825(03)00391-8.

2. Bouchard, P.O.; Bay, F.; Chastel, Y.; Tovena, I. 2000. Crack propagation modelling using an advanced remeshing technique, Computer Methods in Applied Mechanics and Engineering 189(3): 723-742. https://doi.org/10.1016/S0045-7825(99)00324-2.

3. Suna, S.; Sundararaghavana, V. 2016 Modeling Crack Propagation in Polycrystalline Microstructure using Variational Multiscale Method, Mathematical Problems in Engineering (2):1-14. https://doi.org/10.1155/2016/4715696.

4. Barbieri, E.; Petrinic, N. 2014. Three-dimensional crack propagation with distance-based discontinuous kernels in mesh free methods, Computational Mechanics 53(2):325-342. https://doi.org/10.1007/s00466-013-0910-3.

5. Belytschko, T.; Lu, Y.Y.; Gu, L. 1994. Element-free Galerkin methods, International Journal for Numerical Methods in Engineering 37(2):229-256. https://doi.org/10.1002/nme.1620370205.

6. Menouillard, T.; Belytschko T. 2010. Dynamic fracture with mesh-free enriched XFEM, Acta Mechanica 213(1-2):53-69.

https://doi.org/10.1007/s00707-009-0275-z.
7. Wu, J.; Li, F.; Xu, S. 2015. Extended embedded crack finite element method for modeling localized failures in concrete structures, Computer methods in applied mechanics and engineering 285:346-378. https://doi.org/10.1016/j.cma.2014.11.013.

8. Mao, Z.; Liu, G.R.; Dong, X. 2017. A comprehen-sive study on the parameters setting in smoothed particle hydrodynamics (SPH) method applied to hydrodynamics problems, Computers \& Geotechnics 92:77-95. https://doi.org/10.1016/j.compgeo.2017.07.024.

9. Ma, N.; Takada, K.; Okada, H. 2016. A meshfree continuous-discontinuous approach for the ductile fracture modeling in explicit dynamics analysis, Computational Mechanics 58(3):391-409. https://doi.org/10.1007/s00466-016-1299-6.

10. Hajiazizi, M.; Bastan, P. 2014. The elastoplastic analysis of a tunnel using the EFG method: A comparison of the EFGM with FEM and FDM, Applied Mathematics \& Computation, 234(C):82-113. https://doi.org/10.1016/j.amc.2014.02.024.

11. Liu, G.R.; Gu, Y.T. 2005. An Introduction to Meshfree Methods and Their Programming, Springer Netherlands, 97-101. https://doi.org/10.1007/1-4020-3468-7.

12. Krysl, P.; Belytschko, T. 2015. The Element Free Galerkin method for dynamic propagation of arbitrary 3-D cracks, International Journal for Numerical Methods in Engineering, 44(6):767-800. https://doi.org/10.1002/(SICI)10970207(19990228)44:6<767: AID-NME524>3.0.CO;2-G.

13. Belytschko, T.; Krongauz, Y.; Fleming, M.; Organ, D.; Liu, W. 1996. Smoothing and accelerated computations in the element free Galerkin method, Journal of Computational \& Applied Mathematics, 74(1-2):111126. https://doi.org/10.1016/0377-0427(96)00020-9.

14. Organ, D.; Fleming, M.; Terry, T.; Belytschko, T. 1996. Continuous meshless approximation for nonconvex bodies by diffraction and transparency, Computational Mechanics, 18(3):225-235. https://doi.org/10.1007/BF00369940.

15. Tanaka, S.; Suzuki, H.; Sadamoto, S; Yu T.T.; Bui, T.Q. 2016. Accurate evaluation of mixed-mode intensity factors of cracked shear-deformable plates by an enriched meshfree Galerkin formulation, Archive of Applied Mechanics 87(2):1-20. https://doi.org/10.1007/s00419-016-1193-x.

16. Barbier, E.; Petrinic, N. 2013 Multiple Crack Growth and Coalescence in Meshfree Methods with Adistance Function-Based Enriched Kernel, Key Engineering Materials 560:37-60. https://doi.org/10.4028/www.scientific.net/KEM.560.37.

17. Tanaka, S.; Suzuki, H.; Sadamoto, S.; Bui T.Q. 2016. Accurate evaluation of mixed-mode intensity factors of cracked shear-deformable plates by an enriched meshfree Galerkin formulation, Archive of Applied Mechanics, 87(2):1-20. https://doi.org/10.1007/s00419-016-1193-x.

18. Molski, K.L.; Truszkowski, W. 2010. Determination of KI and KII for curvilinear cracks under a non-uniform biaxial stress field, Fatigue \& Fracture of Engineering Materials \& Structures, 19(10):1255-1263. https://doi.org/10.1111/j.1460-2695.1996.tb00948.x. 
19. Chan, S.K.; Tuba, I.S.; Wilson, W.K. 1970. On the finite element method in linear fracture mechanics, Engineering Fracture Mechanics, Engineering Fracture Mechanics 2(1):1-17.

https://doi.org/10.1016/0013-7944(70)90026-3.

Yuantao Sun, Zengzeng Zhang, Qing Zhang, Xianrong Qin

\section{RESEARCH ON CRACK DISCONTINUOUS INTERFACE SIMULATION METHOD BASED ON THE MESHFREE DIFFRACTION CRITERION}

\section{S u m m a r y}

Mesh free method is a new numerical method whose solution domain only discredited into independent nodes. To model crack discontinuous interface in practical, the equivalent distance for the weight function arguments is adapted. Firstly, the paper analysis the feature of the func- tion, which point out that the methods have their own disadvantages. And then the paper put forward new binary linear equation, which is used to the weight function arguments to dispose of the crack's noncontiguous interface. The function can simulate the displacement field around crack tip or crack closure line and also avoid the value overflow phenomenon which the node and a computation superposition occurs in the present processing method. The method has provided new mentality and idea for the present mesh free method in crack analysis. Finally, three typical crack theoretical models are established as example. The comparison with the analytical solution shows that the displacement value the crack and the stress intensity factors has a better precise based on the new method, and also verified the method is valid.

Keywords: mesh free method, crack discontinuous interface, weight function, diffraction criterion.

Received September 10, 2017 Accepted June 14, 2018 